\title{
Sources of bias in synchronization measures and how to minimize their effects on the estimation of synchronicity: Application to the uterine electromyogram
}

\author{
Terrien Jérémy ${ }^{1}$, Marque Catherine², Germain $\mathrm{Guy}^{3}$ and Karlsson Brynjar ${ }^{1,4}$ \\ ${ }^{1}$ Reykjavik University \\ Iceland \\ ${ }^{2}$ Compiègne University of technology \\ France \\ ${ }^{3}$ CRC MIRCen, CEA-INSERM \\ France \\ ${ }^{4}$ University of Iceland \\ Iceland
}

\section{Introduction}

Preterm labor (PL) is one of the most important public health problems in Europe and other developed countries as it represents nearly $7 \%$ of all births. It is the main cause of morbidity and mortality of newborns. Early detection of a PL is important for its prevention and for that purpose good markers of preterm labor are needed. One of the most promising biophysical markers of PL is the analysis of the electrical activity of the uterus. Uterine electromyogram, the so called electrohysterogram (EHG), has been proven to be representative of uterine contractility. It is well known that the uterine contractility depends on the excitability of uterine cells but also on the propagation of electrical activity to the whole uterus. The different algorithms proposed in the literature for PL detection use only the information related to local excitability. Despite encouraging results, these algorithms are not reliable enough for clinical use. The basic hypothesis of this work is that we could increase PL detection efficiency by taking into account the propagation information of the uterus extracted from EHG processing. In order to quantify this information, we naturally applied the different synchronization methods previously used in the literature for the analysis of other biomedical signals (i.e. EEG).

The investigation of the coupling between biological signals is a commonly used methodology for the analysis of biological functions, especially in neurophysiology. To assess this coupling or synchronization, different measures have been proposed. Each measure assumes one type of synchronization, i.e. amplitude, phase... Most of these measures make some statistical assumptions about the signals of interest. When signals do 
not respect these assumptions, they give rise to a bias in the measure, which may in the worst case, lead to a misleading conclusion about the system under investigation. The main sources of bias are the noise corrupting the signal, a linear component in a nonlinear synchronization and non stationarity. In this chapter we will present the methods that we developed to minimize their effects, by evaluating them on synthetic as well as on real uterine electromyogram signals. We will finally show that the bias free synchronization measures that we propose can be used to predict the active phase of labor in monkey, where the original synchronization measure does not provide any useful information. In this chapter we illustrate our methodological developments using the nonlinear correlation coefficient as an example of a synchronization measure in which the methods can be used to correct for bias.

\section{Uterine electromyography}

The recording of the electrical activity of the uterus during contraction, the uterine electromyography, has been proposed as a non invasive way to monitor uterine contractility. This signal, the so called Electrohysterogram (EHG), is representative of the electrical activity occurring inside the myometrium, the uterine muscle. The EHG is a strongly non stationary signal mainly composed of two frequency components called FWL (Fast Wave Low) and FWH (Fast Wave High). The characteristics of the EHG are influenced by the hormonal changes occurring along gestation. The usefulness of the EHG for preterm labor prediction has been explored as it is supposed to be representative of the uterus contractile function.

\subsection{Preterm labor prediction by use of external EHG}

Gestation is known to be a two-step process consisting of a preparatory phase followed by active labor (Garfield \& al., 2001). During the preparatory phase, the uterine contractility evolves from an inactive to a vigorously contractile state. This is associated to an increased myometrial excitability, as well as to an increased propagation of the electrical activity to the whole uterus (Devedeux \& al., 1993; Garfield \& Maner, 2007).

Most studies have focused on the analysis of the excitability of the uterus using two to four electrodes. It is generally supposed that the increase in excitability is mainly observable through an increase in the frequency of FWH (Buhimschi \& al., 1997; Maner \& Garfield, 2007). Some authors, like (Buhimschi \& al., 1997), also used the energy of the EHG as potential parameter for the prediction of preterm labor. This parameter is however highly dependent on experimental conditions like the inter-electrode impedance. A relatively recent paper used the whole frequency content, i.e. FWL + FWH, of the EHG for PL prediction (Leman \& al., 1999). This study, based on the characterization of the timefrequency representation of the EHG, demonstrated that a fairly accurate prediction can be made as soon as 20 weeks of gestation in human pregnancies.

In spite of very exciting results, this method is not currently used in routine practice due to the discrepancy between the different published studies, a strong variability of the results obtained and thus a not sufficient detection ratio for clinical use. Increasingly, teams working in this field tried to increase the prediction ratio by taking into account the propagation phenomenon in addition to the excitability (Euliano \& al., 2009; Garfield \& Maner, 2007). A uterus working as a whole is a necessary condition to obtain efficient 
contractions capable of dilating the cervix and expulsing the baby. The study of the propagation of the electrical activity of the uterus has been performed in two different ways. The first approach consists, like for skeletal muscle, in observing and characterizing the propagation of the electrical waves (Karlsson \& al., 2007; Euliano \& al., 2009). The second one consists in studying the synchronization of the electrical activity at different locations of the uterus during the same contraction by using synchronization measures (Ramon \& al., 2005; Terrien \& al., 2008b). The work presented in this chapter derived from this second approach.

\subsection{Possible origins of synchronization of the uterus at term}

The excitability is mainly controlled at a cellular level by a modification of ion exchange mechanisms. Propagation is mainly influenced by the cell-to-cell electrical coupling (intercellular space, GAP junctions). More precisely, the propagation is a multi-scale phenomenon. At a cellular level, it mainly takes place through GAP junctions (Garfield \& Hayashi, 1981; Garfield \& Maner, 2007). At a higher scale, there is preferential propagation pathways called bundles which represent group of connected cells organized as packet (Young, 1997; Young \& Hession, 1999). The organization of the muscle fibers might also play an important role in propagation phenomenon and characteristic. Contrary to skeletal muscle, the fibers of uterus are arranged according to three different orientations. The role of the nerves present in the uterus is still debated but may be responsible of a long distance synchronization of the organ (Devedeux \& al., 1993).

The recent studies focusing on the propagation characterization used multi electrode grids position on the woman abdomen in order to picture the contractile state of the uterus along the contraction periods. The most common approach uses the intercorrelation function in order to detect a potential propagation delay between the activities of two distant channels. It has been shown that there is nearly no linear correlation between the raw electrical signals (Duchêne \& al., 1990; Devedeux \& al., 1993) so all these studies used the envelope ( instantaneous energy) of the signals to compute propagation delays. Only recently, two studies have used synchronization parameters on the EHG in order to analyze the propagation/synchronization phenomenon involved (Ramon \& al., 2005; Terrien \& al., 2008b).

\section{Synchronization measures}

If we are interested in understanding or characterizing a particular system univariate signal processing tools may be sufficient. The system of interest is however rarely isolated and is probably influenced by other systems of its surrounding. The detection and comprehension of these possible interactions, or couplings, is challenging but of particular interest in many fields as mechanics, physics or medicine. As a biomedical example, we might be interested in the coupling of different cerebral structures during a cognitive task or an epilepsy crisis. To analyze this coupling univariate tools are no longer sufficient and we would need multivariate or at least bivariate analysis tools. These tools have to be able to detect the presence or not of a coupling between two systems but also to indicate the strength and the direction of the coupling (Figure 1). A coupling measure or a synchronization measure has so to be defined. 

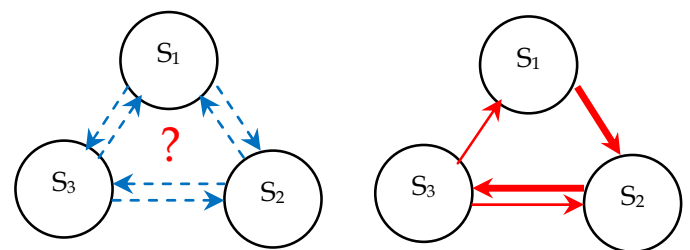

Fig. 1. Schema of synchronization analysis between 3 systems. These methods are able to detect the presence or absence, the strength and the direction of the couplings defining a coupling pattern.

There are a numerous synchronization measures in the literature. The interested reader can find a review of the different synchronization measures and their applications for EEG analysis in (Pereda \& al., 2005). Each of them makes a particular hypothesis on the nature of the coupling. As simple examples, it can be an amplitude modulation or a frequency modulation of the output of one system in response to the output of another one. These measures can be roughly classified according to the approach that they are based on (Table $1)$.

\begin{tabular}{|c|c|}
\hline \hline Approach & Synchronization measure \\
\hline \hline \multirow{2}{*}{ Correlation } & Linear correlation coefficient \\
\cline { 2 - 2 } & Coherence \\
\cline { 2 - 2 } & Nonlinear correlation coefficient \\
\cline { 2 - 2 } Phase synchronization & Phase entropy \\
\hline \multirow{2}{*}{ Generalized synchronization phase coherence } \\
\cline { 2 - 2 } & Synchronization likelihood \\
\hline
\end{tabular}

Table 1. Different approaches and associated synchronization measures.

To this non exhaustive list of measures, we could add two other particular classes of methods. The methods presented Table 1 are bivariate methods. In the case of more than two systems possibly coupled to each other, these methods might give an erroneous coupling pattern. Therefore multivariate synchronization methods have been introduced recently (Baccala \& Sameshima 2001a, 2001b; Kus \& al., 2004). The main associated synchronization measures are the partial coherence and the partial directed coherence. The last class of method is the event synchronization. One example of derived synchronization measure is the Q measure (Quian Quiroga \& al., 2002).

In this work we will treat in more detail the nonlinear correlation coefficient in the context of a practical approach. In our context of treating bias in synchronization measures, we chose this particular measure since in previous study the linear correlation coefficient was not able to highlight any linear relationship between the activity of different part of the uterus during contractions. The methods of correcting for bias presented in this work however allowed us to use this measure to show the real underlying relation in the signals. We however want to stress that the methods presented here can be used with any other synchronization measures. 


\subsection{Linear correlation coefficient}

The linear correlation coefficient represents the adjustment quality of a relationship between two time series $x$ and $y$, by a linear curve. It is simply defined by:

$$
r^{2}=\frac{\operatorname{cov}^{2}(x, y)}{\operatorname{var}(x) \cdot \operatorname{var}(y)}
$$

where cov and var stand for covariance and variance respectively.

This model assumes a linear relationship between the observations $x$ and $y$. In many applications this assumption is false. More recently, a nonlinear correlation coefficient has been proposed in order to be able to model a possible nonlinear relationship (Pijn \& al., 1990).

\subsection{Nonlinear correlation coefficient}

The nonlinear correlation coefficient $\left(H^{2}\right)$ is a non parametric nonlinear regression coefficient of the relationship between two time series $x$ and $y$. In practice, to calculate the nonlinear correlation coefficient, a scatter plot of $y$ versus $x$ is studied. The values of $x$ are subdivided into bins; for each bin, the $x$ value of the midpoint $\left(p_{i}\right)$ and the average value of $y\left(q_{i}\right)$ are calculated. The curve of regression is approximated by connecting the resulting points $\left(p_{i}, q_{i}\right)$ by segments of straight lines; this methodology is illustrated figure 2 . The nonlinear correlation coefficient $H^{2}$ is then defined as:

$$
H_{y / x}^{2}=\frac{\sum_{k=1}^{N} y(k)^{2}-\sum_{k=1}^{N}(y(k)-f(x(k)))^{2}}{\sum_{k=1}^{N} y(k)^{2}}
$$

where $f(x)$ is the linear piecewise approximation of the nonlinear regression curve. This parameter is bounded by construction between $[0,1]$. The measure $H^{2}$ is asymmetric, because $H_{y / x}^{2}$ may be different to $H_{x / y}^{2}$ and can thus gives information about the direction of coupling between the observations. If the relation between $\mathrm{x}$ and $\mathrm{y}$ is linear $H_{y / x}^{2}=H_{x / y}^{2}$ and is close to $r^{2}$. In the case of a nonlinear relationship, $H_{y / x}^{2} \neq H_{x / y}^{2}$ and the difference $\Delta H^{2}$ indicates the degree of asymmetry. $H^{2}$ can be maximized to estimate a time delay $\tau$ between both channels for each direction of coupling. Both types of information have been used to define a measure of the direction of coupling and successfully applied to EEG by (Wendling \& al., 2001). 

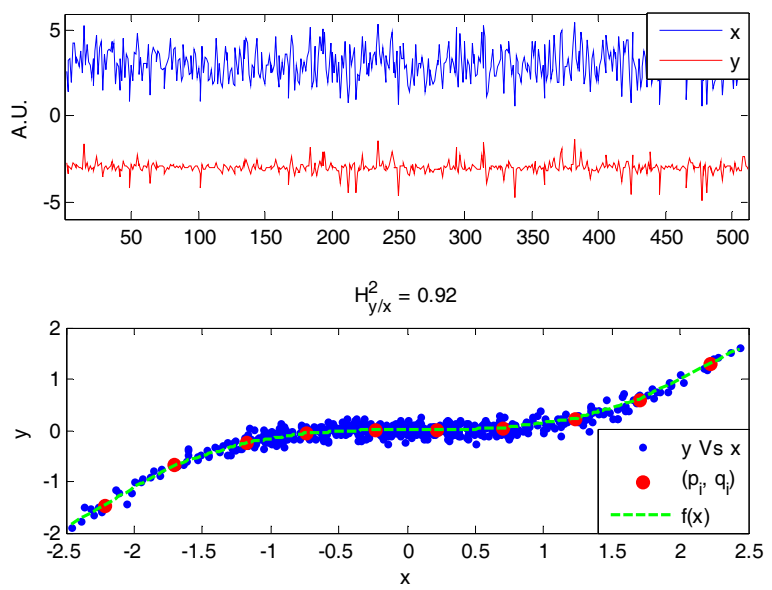

Fig. 2. Original data $x=N(0,1)$ and $y=(x / 2)^{3}+N(0,0.1)$ (upper panel) and construction of the piecewise linear approximation of the nonlinear relationship between $\mathrm{x}$ and $\mathrm{y}$ in order to compute the parameter $\mathrm{H}^{2}$ (lower panel). For comparison, the linear correlation coefficient $\mathrm{r}^{2}$ is only 0.64 .

This method is non parametric is the sense that it does not assume a parametric model of the underlying relationship. The number of bins needs however to be defined in a practical application. Our experience shows that this parameter is not crucial regarding the performances of the method. It has to be set anyway in accordance to the nonlinear function that might exist between the input time series. Similarly to what is expressed by the Shannon theorem, the sampling rate of the nonlinear function must be sufficient to model properly the nonlinear relationship. The limit case of 2 bins might give a value close or equal to the linear correlation coefficient. The hypothetic result that we might obtain with a very high number of bins highly depends on the relationship between the time series. It may tend to an over estimation due to an over fitting of the relationship corrupted by noise. We so suggest evaluating the effect of this parameter on the estimation of the relationship derived from a supposed model of the relationship or clean experimental data.

\section{Effect of noise in synchronization measure}

\subsection{Denoising methods}

Noise corrupting the signals is the most common source of bias. It is present in nearly all real life measurements in varying quantities. The noise can come from the environment of the electrodes and the acquisition system, e.g. powerline noise, electronic noise, or from other biological systems not under investigation like ECG, muscle EMG ... To reduce the influence of this noise on the synchronization measure, one may use digital filters to increase the signal to noise ratio (SNR) expressed in decibel (dB). We have to differentiate linear filters like classical Butterworth filters, and nonlinear filters like wavelet filters. Nonlinear filters are filters that can make the distinction between the signal of interest and the part of the noise present in the same frequency band in order to remove it. With linear filter it is not the case and we have to set the cutting frequency according to the bandwidth 
of the signal of interest. This kind of filter cannot remove the noise present in the signal bandwidth without distorting the signal itself.

In synchronization analysis, only linear filters have been used in the literature to our knowledge. However, linear filters are known to dephase the filtered signal. In order to avoid this distortion, phase preserving filters are used instead. Practically, this is realized by filtering two times the noisy signal, one time in the forward direction and the second time in the reverse direction to cancel out the phase distortion.

\subsection{Example}

To model and illustrate the effect of noise on synchronization measures, we used two coupled chaotic Rössler oscillators. This model has been widely used in synchronization analysis due to is well known behavior. The model is defined by:

$$
\begin{aligned}
& \dot{x}_{1}=-\omega_{1}(t) y_{1}-z_{1} \\
& \dot{y}_{1}=\omega_{1}(t) x_{1}+0.15 y_{1} \\
& \dot{z}_{1}=0.2+z_{1}\left(x_{1}-10\right) \\
& \dot{x}_{2}=-\omega_{2}(t) y_{2}-z_{2}+C(t)\left(x_{2}-x_{1}\right) \\
& \dot{y}_{2}=\omega_{2}(t) x_{2}+0.15 y_{2} \\
& \dot{z}_{2}=0.2+z_{2}\left(x_{2}-10\right)
\end{aligned}
$$

The function $C(t)$ allows us to control the coupling strength between the two oscillators. The system was integrated by using an explicit Runge-Kutta method of order 4 with a time step $\Delta t=0.0078$. For this experiment we used the following Rössler system configuration: $\omega_{1}=$ $0.55, \omega_{2}=0.45$ and $C=0.4$. On the original time series we added some Gaussian white noise in order to obtain the following $\mathrm{SNR}=\{30 ; 20 ; 15 ; 10 ; 5 ; 0\} \mathrm{dB}$. The synchronization analysis was then realized on the filtered version of the noisy signals using a $4^{\text {th }}$ order phase preserving Butterworth filter. The results of this experiment are presented figure 3.

As we can see, the measured coupling drops dramatically for SNR below $20 \mathrm{~dB}$. The filtering procedure is able to keep the measured coupling close to the reference down to 10 $\mathrm{dB}$. For more noise, the measured coupling deviated significantly from the real value due to the non negligible amount of noise inside the bandwidth of the signals. The results obtained with a simple linear filter are surprisingly good. It can be explained by the very narrow bandwidth of the Rössler signals. The amount of noise present in the bandwidth of the signals is very small as compared to the total amount of noise added in the whole frequency band. In this situation, the use of nonlinear filter might be interesting. A study of the possible influences of the nonlinear filtering methods on the synchronization measures has to be done first and might be interesting for the community using synchronization measures. 


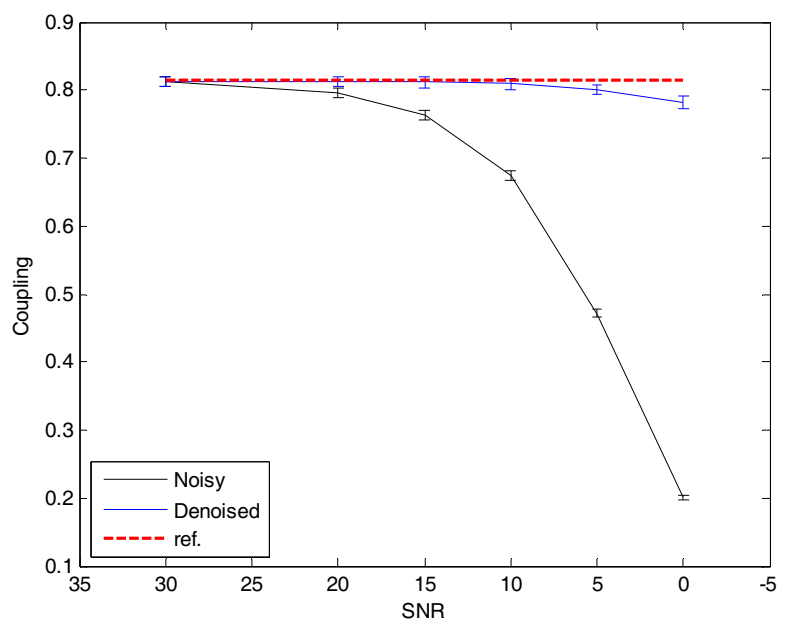

Fig. 3. Evolution of the coupling as a function of the imposed SNR before (Noisy) and after denoising (Denoised). The reference synchronization value is plotted by a horizontal dashed line.

The main physiological noises corrupting external EHG are the maternal skeletal EMG and ECG. The noise and the EHG present overlapping spectra. A specific nonlinear filter has been developed for denoising properly these EHG (Leman \& Marque, 2000). Internal EHG, like the signals used here, are less corrupted and allow the use of classical phase preserving linear filters. An analysis of the possible effects of this type of denoising will have to be done for an application of synchronization analysis of external EHG, as it is performed on pregnant women.

\section{Nonlinearity testing with surrogate measure profile}

To test a particular hypothesis on a time series, surrogate data are usually used. They are built directly from the initial time series in order to fulfill the conditions of a particular null hypothesis. One common hypothesis is the nonlinearity of the original time series. The procedure involves the analysis of the statistics of the surrogates as compared to the statistic found with the original data in order to define its z-score. The z-score assumes that the surrogate measure profile presents a Gaussian distribution. If this is not the case the test might be erroneous.

We propose to use a surrogate corrected value instead of the z-score of a particular statistic. We also derive a statistical test based on the fitting of the surrogate measure profile distribution. We demonstrate the proposed method on the nonlinear correlation coefficient $\left(H^{2}\right)$ as the initial statistic. The performance of the corrected statistic was evaluated on both synthetic and real EHG signals. 


\subsection{Surrogate data}

Surrogate data are time series which are generated in order to keep particular statistical characteristics of an original time series while destroying all others. They have been used to test for nonlinearity (Schreiber \& Schmitz, 2000) or nonstationarity (Borgnat \& Flandrin, 2009) of time series for instance. The classical approaches to constructing such time series are phase randomization in the Fourier domain and simulated annealing (Schreiber \& Schmitz, 2000). Depending on the method used to construct the surrogates, a particular null hypothesis is assumed. The simulated annealing approach is very powerful since nearly any null hypothesis might be chosen according to the definition of an associated cost function. As a first step, we chose the Fourier based approach.

The Fourier based approach consists mainly in computing the Fourier transform, F, of the original time series $x(t)$.

$$
X(f)=F\{x(t)\}=A(f) e^{i \Phi(f)}
$$

where $\mathrm{A}(\mathrm{f})$ is the amplitude and $\Phi(\mathrm{f})$ the phase. The surrogate time series is obtained by rotating the phase $\Phi$ at each frequency $f$ by an independent random variable $\varphi$ taking values in the range $[0,2 \pi)$ and going back to the temporal domain by inverse Fourier transform $\mathrm{F}^{-1}$, that is:

$$
\tilde{x}(t)=F^{-1}\{\tilde{X}(f)\}=F^{-1}\left\{A(f) e^{i[\Phi(f)+\varphi(f)]\}}\right.
$$

By construction, the surrogate has the same power spectrum and autocorrelation function as the original time series but not the same amplitude distribution. This basic construction method has been refined to assume different null hypothesis. We used the iterative amplitude adjusted Fourier transform method to produce the surrogates (Schreiber \& Schmitz, 2000). Basically, this iterative algorithm starts with an initial random shuffle of the original time series. Then, two distinct steps will be repeated until a stopping criterion is met, i.e. mean absolute error between the original and surrogate amplitude spectrum. The first step consists in a spectral adaptation of the surrogate spectrum and the second step in an amplitude adaptation of the surrogate. At convergence, the surrogate has the same spectrum and amplitude distribution of the original time series, but all nonlinear structures present in the original time series are destroyed.

\subsection{Use of surrogate measure profile}

On each surrogate $j$ we can compute a measure $\Theta_{0}(j)$. All values of $\Theta_{0}(j)$ form what we call a surrogate measure profile $\Theta_{0}$. Surrogate measure profiles $\Theta_{0}$ are usually used in order to give a statistical significance to a measure $\Theta_{1}$ against a given null hypothesis $H_{0}$. The classical approach assumes that $\Theta_{0}$ is normally distributed and uses the z-score. The empirical mean $\left\langle\Theta_{0}>\right.$ and standard deviation $\sigma\left(\Theta_{0}\right)$ of $\Theta_{0}$ are calculated. The $z$-score of the observed value $\Theta_{1}$ is then:

$$
z=\left|\frac{\Theta_{1}-\left\langle\Theta_{0}\right\rangle}{\sigma\left(\Theta_{0}\right)}\right|
$$

The hypothesis test is usually considered as significant at a significance level $p<0.05$ when $z$ $\geq 1.96$. The z-score has been also directly used to measure the nonlinearity of a univariate or a multivariate system (Prichard \& Theiler, 1994).

In practice, the normality assumption should be checked before using the $z$ statistic. For that purpose, the Kolmogorov-Smirnov or Lilliefors test might be used. The KolmogorovSmirnov test uses a predefined normal distribution of the null hypothesis, i.e. known mean 
and variance. The Lilliefors test is on the contrary based on a mean and variance of the distribution derived directly from the data.

\subsection{Percentile corrected statistic and associated hypothesis test}

The distributions of $\Theta_{0}$ might be non Gaussian as attested by a Lilliefors test for example. In that case, the use of $z$-score statistics may be erroneous or at least meaningless. We propose to use instead a measure corrected according to the statistics of the surrogates. This measure, $\Theta_{c x}$, is defined as:

$$
\Theta_{c x}=\Theta_{1}-P_{x}\left(\Theta_{0}\right)
$$

where $P_{x}(y)$ stands for the $x^{\text {th }}$ percentile of the data $y$.

The study of the statistical distribution of $\Theta_{0}$ allows us to define a statistical test even when dealing with non Gaussian distributions. In practice, we have noticed that the distribution of $\Theta_{0}$ follows approximately a Gamma law $\Gamma(a, \beta)$ when the distribution is not Gaussian. A distribution model can be fitted directly on the surrogate data by maximum likelihood estimation. This model allows us to easily define a statistical threshold for a given probability $p$, over which the observed value $\Theta_{1}$ is considered as significant. The inverse of the Gamma cumulative distribution function, parameterized by the fitted $a$ and $\beta$, gives the threshold knowing the chosen probability $p$.

In the context of using the nonlinear correlation coefficient $H^{2}$, we called the corrected measure $\Theta_{c x}, H_{c x}^{2}$ or surrogate corrected nonlinear correlation coefficient. This statistic is bounded between $[-1,1]$ where the sign roughly indicates a non significant test if the percentile $x$ and the probability $p$ coincide. According to the characteristics of the generated surrogate data in this study, the parameter $H_{c x}^{2}$ represents the part of the original $H^{2}$ value unexplained by the linearity presents in the original time series.

From a practical point of view, the only parameter that has to be tuned is the number of surrogates used to construct the surrogate measure profile. This number must be large enough for a good estimation of the density function. It varies largely from one signal to another. The counterparts of choosing a very high number of surrogates is the time of computation especially with long original time series. After empirical evaluation of this parameter, we found that 10000 surrogates was a good compromise for our signals.

\subsection{Results on synthetic signals}

For this experiment we used the following Rössler system configuration: $\omega_{1}=0.55$ and $\omega_{2}=$ 0.45 . The sampling rate was $256 \mathrm{~Hz}$.

An instance of the coupled Rössler systems, with $C=0.5$, is presented figure 4 as well as the corresponding surrogates measure profile. We can clearly see that the original synchronization value $H_{y / x}^{2}$ is above the imposed coupling value $C$. The relatively high values of the measure obtained with the surrogates suggest that a non negligible amount of the observed synchronization value is due to a linear component between the systems. 

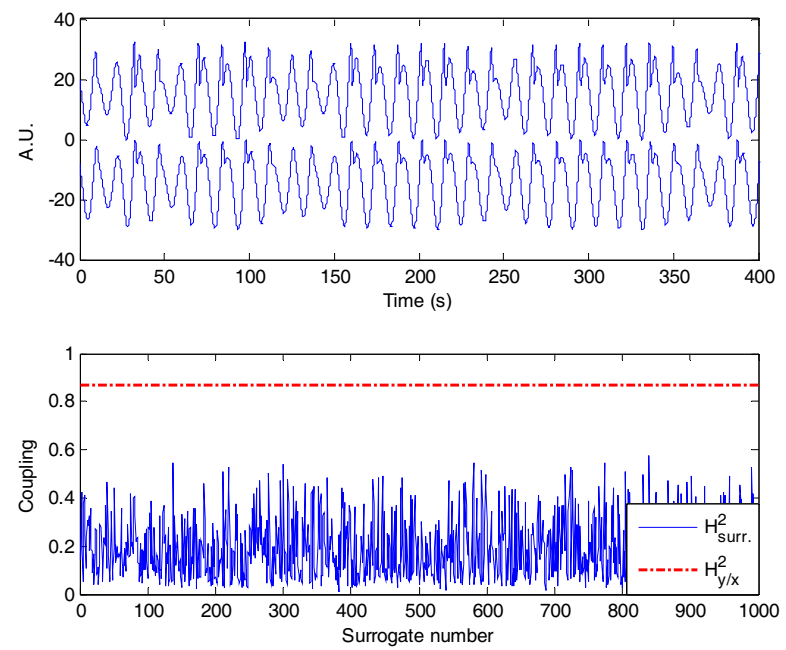

Fig. 4. Example of the output of the model for $C=0.5$ (top panel) and surrogates measure profile (bottom panel).

The distribution of the surrogate profile is depicted figure 5 . We can easily see that the distribution is highly non Gaussian and is best fitted by a Gamma law. A statistical test based on the z-value might thus be erroneous. The non Gaussianity was attested by a Lilliefors test applied on the experimental data. The 90 percentile derived from the fitted law was 0.38 . The measured coupling, 0.87 as observed figure 4 , is above the 90 percentile and thus attests of significant test. The proposed corrected measure, $H_{c x}^{2}$, is in this case 0.49 which is closer to the imposed coupling value $C=0.5$ than the original measure.

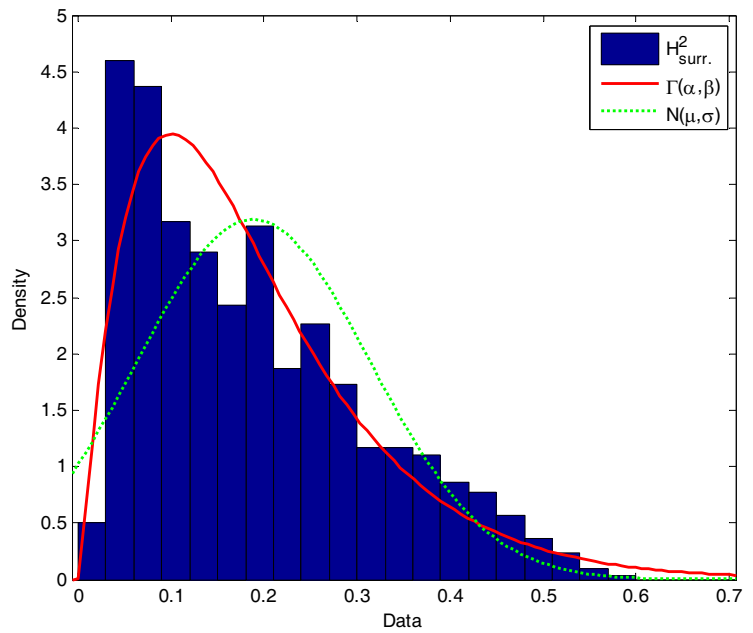

Fig. 5. Distribution of the surrogate values $\left(\Theta_{0}\right)$, Gamma law model $(\Gamma(\alpha, \beta)$, continuous line $)$ and normal law model $(\mathrm{N}(\mu, \sigma)$, dotted line). 
The original synchronization values were always above the imposed coupling (Figure 6). For moderate couplings, below 0.5 , the proposed correction gives nearly identical values as the imposed coupling. From a coupling of 0.5 , the proposed correction underestimates the coupling strength between the systems. More importantly, we can notice that the difference between the original and the corrected values is nearly constant. It indicates that the nature of the relationship between the Rössler systems is identical whatever the imposed coupling strength. This might explain the underestimation of the corrected synchronization due to a "saturation" of the original synchronization at values near 1 .

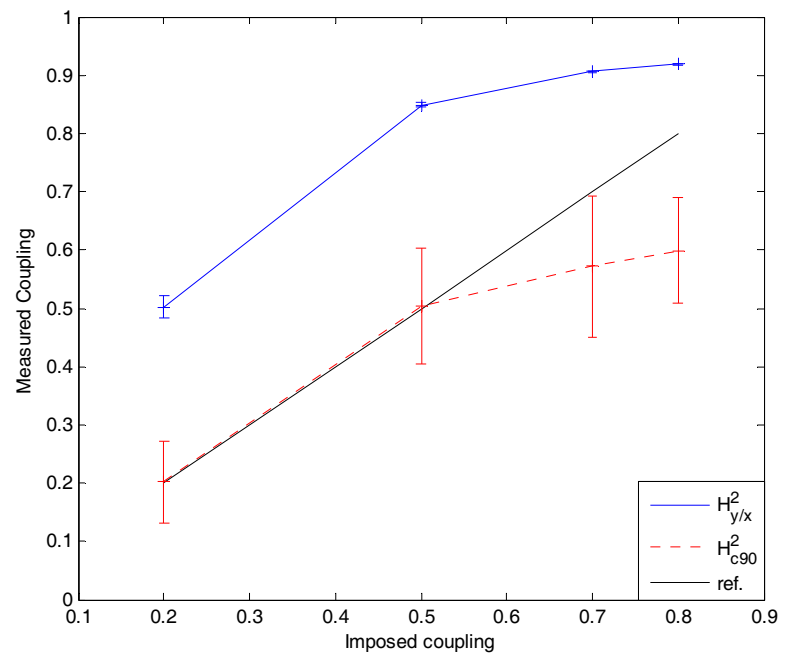

Fig. 6. Original and corrected $H^{2}$ estimations $(\mu \pm \sigma)$ for different imposed coupling values.

\subsection{Results on real EHG signals}

Uterine EMG was recorded on a monkey during labor. Two bipolar channels were sutured on the uterus approximately $7 \mathrm{~cm}$ apart. The two EMG channels were digitalized simultaneously at $50 \mathrm{~Hz}$. A detailed description of the experimental setup can be found in (Terrien \& al., 2008a). The EMG signals were then segmented manually to extract segments containing uterine contractions. The different segments were then band-pass filtered (1 - 4.7 $\mathrm{Hz}$ ) to extract $\mathrm{FWH}$ according to (Devedeux \& al., 1983) by a $4^{\text {th }}$ order phase preserving Butterworth filter. We also showed that a time delay of EHG bursts highlight the synchrony between the signals (Terrien \& al., 2008b). The time delay between bursts that we chose corresponds to the delay needed to maximize the cross-correlation function.

When applied to uterine EMG, we noticed very different behavior of $H^{2}$ in pregnancy and labor contractions as depicted figure 7. In this example, even if the two contractions present nearly the same original synchronization values $(0.13$ and 0.15$)$, their surrogate measure profiles are very different. For the labor contraction, the synchronization measures obtained on surrogates are very low when compared to the original value contrary to the pregnancy EMG, where some surrogates present synchronization measure above the original one. This 
may indicate a strong relationship between the nonlinear components of the EMG burst during labor which seems to be absent or less important during pregnancy. We consider these differences to be useful in differentiating labor and pregnancy contractions. Concerning the statistical test all labor contractions presented a significant test. For pregnancy contractions, the majority but not all the contractions did not test as significant.
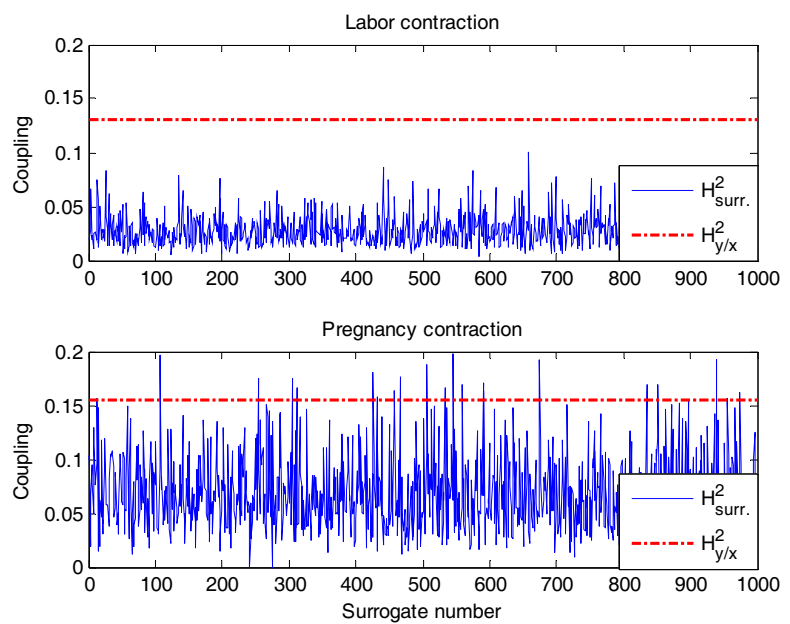

Fig. 7. Example of surrogates measure profile obtained with a labor contraction (top panel) and a pregnancy contraction (bottom panel).

\subsection{Discussion}

Surrogates are constructed to fulfill all characteristics of a null hypothesis that we want to evaluate on a time series. The statistical tests of the considered hypothesis use the $z$ score which implicitly assumes the Gaussianity of the surrogate statistic distribution. In case of non Gaussian statistics, the usual test might fail or simply gives rise to erroneous conclusion. We proposed to use, instead of the z-score, a percentile corrected statistic. This corrected value is thought to be independent of the surrogate distribution. We derived a statistical test by simply fitting the surrogate distribution by a given distribution model and defining a statistical threshold. We demonstrated the satisfactory use of the proposed approach on synthetic and real signals as well. When applied to the nonlinear correlation coefficient, we showed that the statistics of the surrogate measure profile present a Gamma distribution, probably explained by the quadratic nature of the original statistic. For this particular statistic, the new value represents the part of the original value not explained by the linearity present in the original time series. The usefulness of this new measure has of course to be confirmed and tested on different type of data usually used in the field, EEG for example.

The use of this "new" synchronization measure on uterine EMG helped us to show two different behaviors of contractions. We think that this difference might help us in differentiating inefficient (pregnancy) and efficient (labor) contractions in the final aim of labor prediction. This difference in behavior might be explained by the increase in the nonlinearity of the EHG as labor approaches (Radhakrishnan \& al., 2000). The surrogates 
used in this study are also a stationarized version of the original time series. In the case of uterine EMG, we assumed that the EMG bursts were stationary and we imputed the difference between pregnancy and labor to a change in linearity only. Without testing this stationary assumption, we could not be sure about the origin of the observed differences, i.e. linearity or stationarity. The use of surrogates which preserve the non stationarity of the original time series might be helpful for that purpose (Schreiber \& Schmitz, 2000). As a first way to answer this open question, we decided to study the influence of the non stationarity in synchronization analysis and to propose an approach able to take into account this information which is another source of bias of synchronization measure.

\section{Dealing with non stationary signals}

Most synchronization measures are only reliable in the analysis of long stationary time series. A stationary signal is a signal which has all statistical moments constant with time. This strong assumption might be relaxed since this property is impossible to verify. This relaxed condition is called "weak stationarity" of order $n$. A weak stationary signal of order $\mathrm{n}$ presents all moments up to $\mathrm{n}$ that do not vary with time. The stationarity of order 2 is often used (Blanco \& al., 1995).

Many biological signals are however highly non stationary. Nevertheless, the coupling analysis of these non stationary signals is usually performed by using a sliding window in which the signals of interest are supposed to be stationary, or by directly using time dependant synchronization measures like time-frequency approach (Ansary-Asl \& al., 2006). The most commonly used approach is the windowing method. The length of the window has to be set according to the characteristics of the signal of interest. A bad choice of this parameter might have dramatic effects on the obtained results. We propose to use instead a pre-processing step able to detect automatically the longer stationary segments of a signal of interest. This approach avoids making any trade off between the length of the segments and the stationary assumption.

\subsection{The windowing approach}

The windowing approach consists in computing the synchronization parameter in a window of finite length $L$, supposed to be the minimal stationary length of the signals of interest, and shifting the window by a time $\tau$ before computing another value. The time shift is often expressed as a percentage of overlapping between successive windows. The main problem of this method is the estimation of the minimal stationary length.

A tradeoff between the length of the analysis window and the stationary assumption has to be made. The length of the window also limits the accuracy of the time detection of abrupt changes that can reflect biological mechanisms in the underlying systems. As it can be seen figure 8, an increase in the length of the analysis window reduces the variance of the estimation but at a same time smoothes the boundary of the transition times, located in this example roughly at 204 and $460 \mathrm{~s}$. The length of the window is thus an important parameter which has to be set according to a prior knowledge of the minimal length of the stationary parts of the signals or by trial and error. 

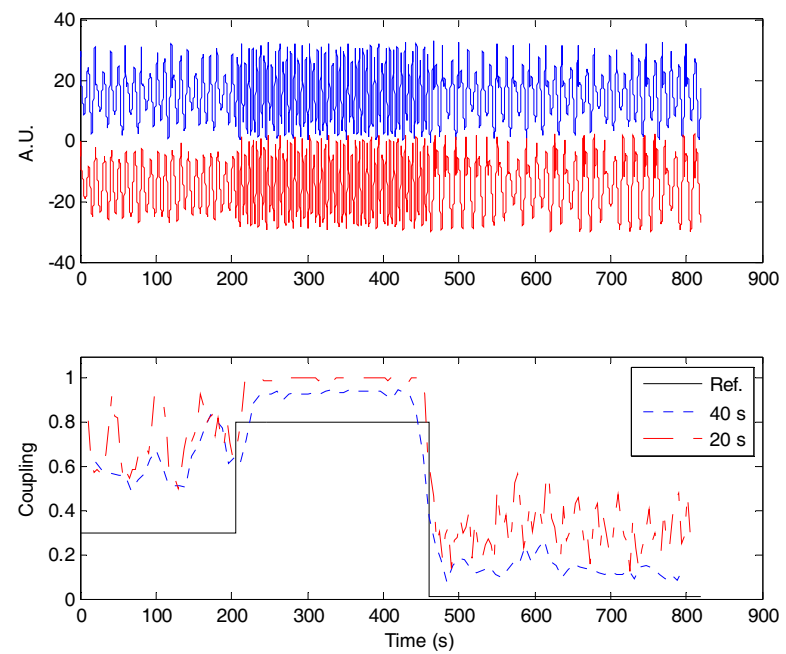

Fig. 8. Example of the output of the Rössler system (top panel) and the corresponding synchronization analysis using $\mathrm{H}^{2}$ (bottom panel) obtained by the windowing approach for a window length of $40 \mathrm{~s}$ or $20 \mathrm{~s}$. The coupling function $C(t)$ is presented as a continuous line (Ref.).

\subsection{Piecewise stationary pre-segmentation (PSP) approach}

Piecewise stationary segmentation algorithms are designed to detect all local stationary partitions composing a signal of interest. They are different from event segmentation algorithms which are designed to detect events of interest, stationary or not, inside a signal. They are mostly based on the analysis of the local statistical properties of the signal. In the context of synchronization analysis, we used advantageously one of these algorithms in order to detect the longer stationary parts inside the signals of interest before applying the traditional synchronization measure. Its results in a succession of windows of automatically locally adapted length. We call this pre-processing step: Piecewise stationary presegmentation or PSP.

The PSP algorithm has been proven to be useful as pre-treatment of synchronization analysis (Terrien \& al., 2008b). In the case of different stationarity changes in the two channels, the univariate PSP (uPSP) method, described in (Terrien \& al., 2008b), might fail to detect these changes. It is explained by the nature of this algorithm which only uses one of both channels for the segmentation. In order to be able to deal properly with this situation, we slightly modified the uPSP algorithm. Instead of using the auto spectrum of only one channel, the stationarity changes are detected using the cross spectrum, thus taking into account the statistical changes in both channels at the same time. We called this method bivariate PSP or bPSP for short. This algorithm, also based on the algorithm developed by Carré and Fernandez (Carré \& Fernandez, 1998), can be described briefly as follows: 
1. Decompose the signal $x$ and $y$ into successive dyadic partitions up to chosen decomposition level $L+1$

2. Compute and denoise, by undecimated wavelet transform, the log cross spectrum of each partition

3. Compute a binary tree of spectral distances between adjacent partitions

4. Search for the tree which minimizes the sum of the spectral distances by a modified version of the best basis algorithm of Coifman Wickerhauser

5. Apply the post processing steps described in (Carré \& Fernandez, 1998) to deal properly with non dyadic partitions

The post processing steps consist mainly in applying the step 1 to 4 on each non terminal node with one level of decomposition and using the original best basis algorithm.

Our modified version of the best basis algorithm differs from the original one only by the node selection rule. This modification was necessary to differentiate the increase in the spectral distances due to the bias of the estimator, or due to signal symmetry around the considered cutting point. Each node $n_{i, j}$ has a cost, corresponding to the spectral distance, $c_{i, j}$. The classical decision rule concerning the selection of a father node $c_{i, j}$ is:

$$
\begin{aligned}
& \text { if }\left(c_{i, j} \leq c_{i+1,2 j}+c_{i+1,2 j+1}\right) \text { then } \\
& \text { Mark the node as a part of the best basis } \\
& \text { else } \\
& \text { endif } \quad c_{i, j}=c_{i+1,2 j}+c_{i+1,2 j+1}
\end{aligned}
$$

The empirical modification of the selection rule is simply a. $c_{i, j} \leq c_{i+1,2 j}+c_{i+1,2 j+1}$ with $a>2$. We chose $a=2.5$.

We showed that the use of the bPSP method avoids an arbitrary choice of the channel to which the stationary segmentation is based on and takes in to account the non stationarity of both signals present.

In a practical point of view, the parameters used in this method are mainly the number of decomposition levels in the segmentation procedure and in the wavelet denoising. These parameters are independent. The first one controls the minimal stationary length that the algorithm can detect. It must be roughly adapted to the signal of interest. A too high number of levels might increase the spectral estimation error, and lead to bad segmentation, due to an increased bias of the cross periodogram. The second parameter controlling the denoising of the spectra might lead to over smoothing of the estimated spectra and thus miss some important features in the different local stationary zones.

\subsection{Results on synthetic signals}

The configuration of the Rössler system used in this study is summarized table 2. The sampling rate used was $10 \mathrm{~Hz}$.

\begin{tabular}{cccc}
\hline \hline Time $\mathrm{t}(\mathrm{s})$ & $\omega_{1}(\mathrm{t})$ & $\omega_{2}(\mathrm{t})$ & $\mathrm{C}(\mathrm{t})$ \\
\hline $0-204.8$ & 0.65 & 0.55 & 0.3 \\
$204.8-307.2$ & 1.2 & 0.55 & 0.01 \\
$307.2-460.8$ & 1.2 & 1.1 & 0.8 \\
$460.8-563.3$ & 0.65 & 1.1 & 0.01 \\
$563.3-819.1$ & 0.65 & 0.5 & 0.5 \\
\hline
\end{tabular}

Table 2. Parameters of the coupled Rössler system. 
Figure 9 presents one example of the synthetic signals used and the corresponding synchronization analyses with $H^{2}$. The results obtained by the windowing approach show a synchronization pattern that approximately follows the coupling function. Important differences can be found during periods of low coupling between the two signals. Increasing the length of the window allowed us to significantly decrease the amplitude of the variations of the parameter but at the same time the boundaries of the different coupling periods become smoother. The bPSP approach shows marked transitions between the different coupling periods with relatively constant parameter values. More importantly, the algorithm is able to detect the change points situated at 307.2 and $563.3 \mathrm{~s}$. This time instant corresponds to changes occurring in the second signals. The previous algorithm, presented in (Terrien \& al., 2008b), would not have detected this transition, when using the top signal as reference, and not the transitions at 205 and 470 seconds when using the lower signal as reference. The differences between the coupling function $C(t)$ and the estimates are due to the intrinsic bias of $H^{2}$ as already highlighted in figure 6 of the paragraph 5 .
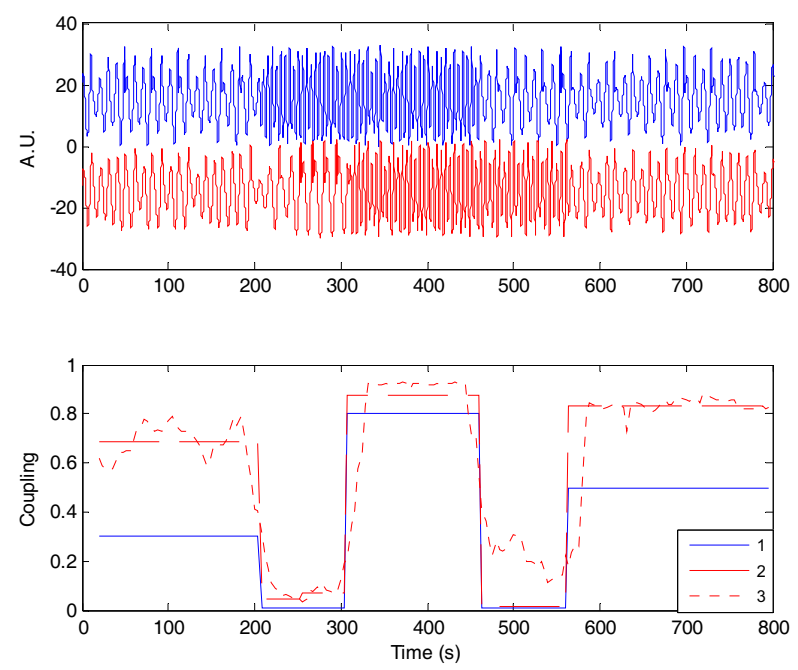

Fig. 9. Example of the output of the Rössler system (top panel) and the corresponding synchronization analysis using $H^{2}$ (bottom panel) obtained by the bPSP (2) and the windowing approach for a window length of $40 \mathrm{~s}(3)$. The coupling function $\mathrm{C}(\mathrm{t})$ is presented as a continuous line (1).

We might be interested in the robustness of a particular method or algorithm in order to apprehend its behavior in the presence of noise. This step is important since most biological signals are very noisy. The main parameters used in robustness analysis are the bias and the variance of the estimator.

We evaluated the robustness of the segmentation algorithm by Monte-Carlo simulations. For different noise (Gaussian white noise) levels, as express by the SNR, the bias and variance of the estimators were computed against the parameter values computed in the reference segments (segments that we would have obtained with a perfect segmentation). 
This methodology allowed us to take into account the intrinsic bias of the synchronization measure.

The robustness analysis (bias and variance) for the parameters $H^{2}$ is presented figure 10 . The stationary approach presents a lower bias than the windowing approach whatever the noise level. The variance obtained by the bPSP method is however greater. The analysis of the individual results showed that this high variance is mainly due to an over segmentation of each stationary zone.
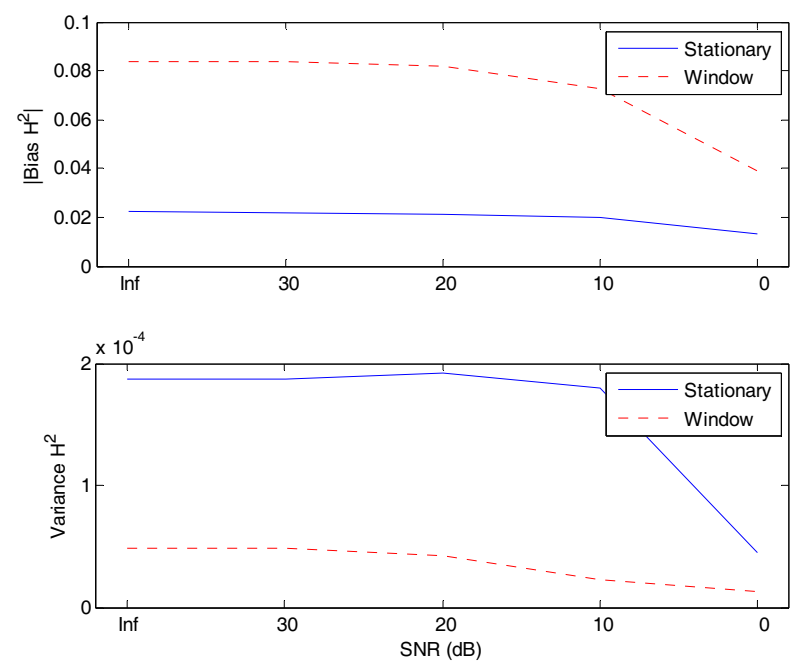

Fig. 10. Absolute value of the bias (top panel) and variance (bottom panel) obtained with the bPSP (continuous line) and the windowing approach (dotted line) for the parameter $\mathrm{H}^{2}$.

\subsection{Results on real EHG signals}

The results of the segmentation of a broad band and narrow band contractile event recorded during labor are presented figure 11 . We can clearly see, on both types of signals, that the algorithm is able to take into account changes occurring in both channels. 

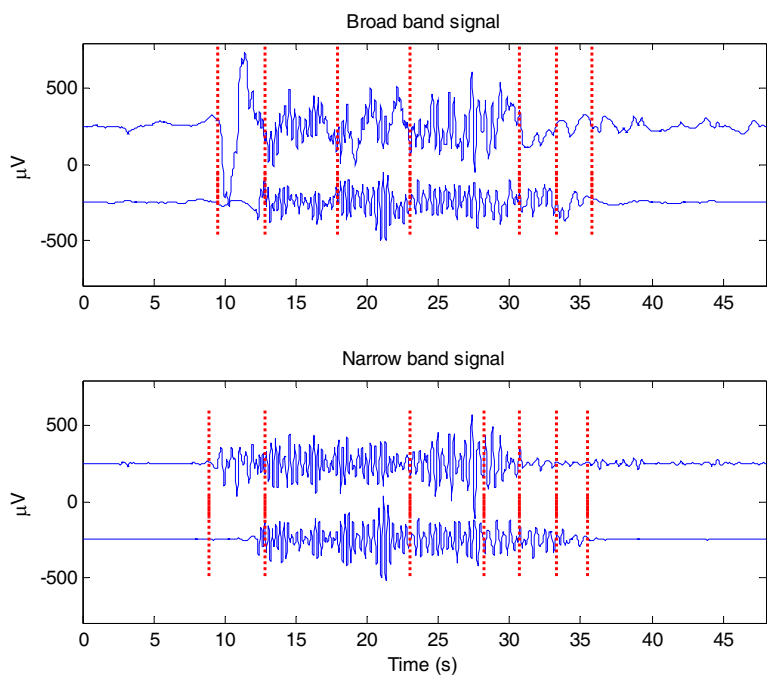

Fig. 11. Example of an electrical contraction burst recorded during the same contraction occurring in labor and their segmentation considering the broad band signals, raw signals (top panel), or a narrow band version of them, FWH filtered (bottom panel).

The results obtained with $H^{2}$ are presented figure 12. On the non shifted broad band signal the values of $H^{2}$ observed inside the contractile event are similar to those observed during the base lines (non EMG segments present before $10 \mathrm{~s}$ and after $35 \mathrm{~s}$ ). The time shift of the signal of the second channel to compensate for the propagation delay of the contraction does not change the parameter pattern significantly. Only the first base line presents lower synchronization values as compared with no time shift. With the narrow band version of the signals, an increase in the parameter $H^{2}$ is clearly observable inside the contractile event when compared to the base line segments. The base lines still present relatively high values. The time shift of $0.18 \mathrm{~s}$ of the second signal causes a strong decrease in the base line values, while the values inside the contractile event increase or stay high. The effects of the time shift is less clear on the narrow band signal maybe due to the short delay between the two channels.

Looking at the results obtained with the windowing approach, no specific pattern can be observed in the same conditions. Moreover, the base lines present stronger or similar values of synchronicity than inside the contractions whatever the considered situation. Similar results were obtained for the other contractions tested. 

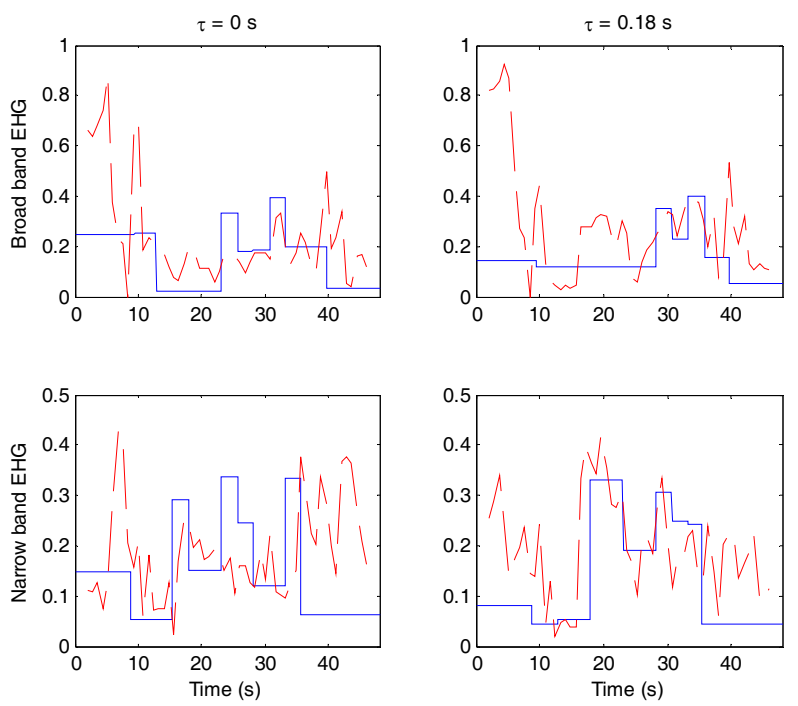

Fig. 12. $H^{2}$ profile obtained on the broad band (first line) or the narrow band (second line) signal of the contractile event presented figure 11 and for no time shift (first column) or a time shift of $\tau=0.18 \mathrm{~s}$ (second column). The results obtained with the windowing approach in the same conditions are plotted with dashed lines.

\subsection{Discussion}

Most physiological signals are non stationary. Their characterization is therefore often difficult. In the context of synchronization analysis, the most commonly used approach is the windowing of the signals of interest before computing the different synchronization measures. The length of the analysis window is however an important parameter and controls the trade-off between stationary assumption of the signals in the window and the accuracy of the analysis. This parameter is often chosen as constant in time. We presented the advantages of using an automatic segmentation procedure of the signal that search for the longer locally adapted stationary parts in the context of synchronization analysis.

The bPSP approach shows reduced bias of the estimators when compared to the reference segmentation. The obtained variance is however higher than with the windowing approach due to over segmentation of the different stationary parts. A better control of the partition fusion procedure in the algorithm might allow us to reduce this over segmentation. The fusion procedure is based on a modification of the Coifman Wickerhauser algorithm, controlled by the parameter $a$. The adaptation of this parameter to the signal of interest might reduce this problem. The length of the minimal stationary zone is dependant of the levels of decomposition. Even if this parameter can be adapted to the signal of interest, a too high number of decomposition levels increases the bias of the spectral estimation due to the shortness of the analyzed data segments. This can introduce errors in the detection of the stationary parts of the signals. The use of other algorithms, which perform segmentation in a continuous time, can be a solution if precise time detection is needed. We have shown that 
the PSP method can be applied to signals with different characteristics and that it gives satisfactory results when compared to the ones obtained with the windowing approach.

Specifically for real EHG, the numerous segments found on each analyzed burst confirm the high non stationarity of the EHG signal, even when band-pass filtered. This might indicate that the difference of surrogate measure profile characteristics between contractions is due to a difference in non stationarity rather than of nonlinearity, even if we cannot exclude definitively or totally this latter hypothesis. Both influences might coexist and be independent. More investigations are thus necessary in order to obtain a clear answer.

We have shown that the use of this method can clearly identify the proper treatment, filtering or time shift for example, needed to identify and highlight synchronization between different parts of the uterus during labor. It might be of use to monitor the evolution of the synchronization of the uterus from pregnancy to labor. This approach can also be used in the determination of the optimal synchronization measure for the uterine EMG.

\section{Labor prediction}

\subsection{ROC curve analysis}

In order to evaluate the possible use of the proposed parameters for the prediction of labor in monkey, we used the classical Receiver Operating Characteristic (ROC) curves. A ROC curve is a graphical tool permitting to evaluate a binary, i.e. two classes, classifier. A ROC curve is the curve corresponding to TPR (True Positive Rate or sensitivity) vs. FPR (False Positive Rate or 1 - Specificity) obtained for different parameter thresholds. ROC curves are classically compared by mean of the Area Under the Curve (AUC) and accuracy (ACC). The AUC was estimated by the trapezoidal integration method. We additionally used the Matthew's Correlation Coefficient (MCC) defined as:

$$
M C C=\frac{T P^{*} T N-F P^{*} F N}{\sqrt{(T N+F N)(T N+F P)(T P+F N)(T P+F P)}}
$$

where TP, TN, FP and FN stand respectively for True Positive, True Negative, False Positve and False Negative values.

\subsection{Prediction using proposed corrected parameters}

Under the hypothesis that the uterus synchronizes as labor approaches, we evaluated the potential of the nonlinear correlation coefficient as a predictor of labor on monkey. We evaluated the performance of the proposed parameters for labor prediction on a data set containing 35 pregnancy and 34 labor contractions. We compared the predictive capability of the nonlinear correlation coefficient $\left(\mathrm{H}^{2}\right)$ and of the 90 percentile corrected nonlinear correlation coefficient, $H_{c 90}^{2}$. We have shown that the segmentation into piecewise stationary parts of EHG highlights synchronicity inside EHG burst, but gives rise to multiple values of $\mathrm{H}^{2}$ within one burst (one for each stationary segment). For comparison purpose, we thus decided to also include the integral of the $\mathrm{H}^{2}$ profile during the bursts, $\int H^{2}$, as a synchronization parameter for labor prediction.

The average results obtained on our data set with the parameter $H_{c 90}^{2}$ and $H^{2}$ are presented table 3. We can see that the original values of $H^{2}$ are very similar or slightly lower during 
labor. This is in contradiction to what should be expected. Indeed, it is assumed that disorganized pregnancy contractions evolve into effectively synchronized labor contractions. The proposed $H_{c 90}^{2}$ synchronization value demonstrates a relatively important increase from pregnancy to labor, by a factor of nearly 10 . The corrected measure is able to differentiate pregnancy and labor contractions and highlights the increase in synchronicity from pregnancy to the active phase of labor. The other synchronization value, $\int H^{2}$, shows a relative increase from pregnancy to labor by a factor of nearly 1.5 (table 3 ). The standard deviation obtained with this parameter is moreover relatively high in both situations. This corrected measure seems also to be able to differentiate pregnancy and labor contractions in spite of the high standard deviation obtained and a lower difference between pregnancy and labor.

\begin{tabular}{ccc}
\hline \hline Parameter & Pregnancy & Labor \\
\hline$H^{2}$ & $0.1377 \pm 0.05$ & $0.1111 \pm 0.02 \dagger$ \\
$H_{c 90}^{2}$ & $0.0036 \pm 0.02$ & $0.0310 \pm 0.02 \ddagger$ \\
$\int H^{2}$ & $3.0995 \pm 1.02$ & $4.7104 \pm 1.12 \ddagger$ \\
\hline
\end{tabular}

Table 3. Mean synchronization measures along gestation ( $\ddagger$ and $\dagger$ indicate a significant difference at $\mathrm{p}=0.01$ and 0.05 respectively, Wilcoxon rank sum test).

The values obtained during pregnancy with $H^{2}$ were higher during pregnancy than during labor. This might indicate a decrease in the synchronization of the uterus in labor. Even if we have considered this option, the ROC curve obtained depicts a bad predictive parameter (Figure 13). Indeed, the first half of the curve lies on the diagonal, which is representative of a random decision. $H_{c 90}^{2}$ increases, as well as $\int H^{2}$, during labor. The ROC curves obtained are close together as depicted figure 13.

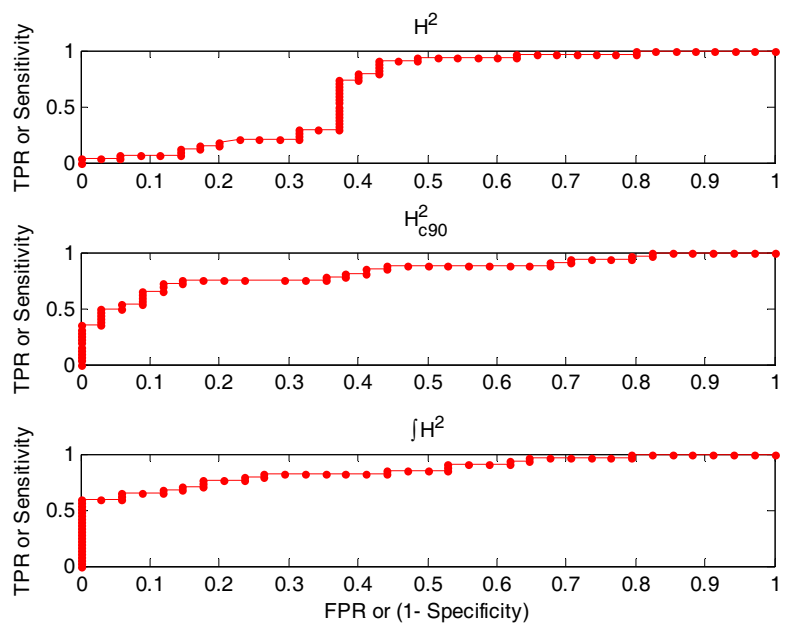

Fig. 13. ROC curve obtained with original, corrected $H^{2}$ measure with surrogates and integral of $H^{2}$ after piecewise stationary segmentation for the prediction of labor. 
A summary of the ROC curves characteristics is presented table 4 . The table indicates that both proposed parameters are better than the original parameter $H^{2}$. The performance values of the parameters $H_{c 90}^{2}$ and $\int H^{2}$ are very close. The main difference between the predictors concerns the first part of the curves, which might indicate a better separability between pregnancy and labor contractions by using the $\int H^{2}$ parameter. We can see for this parameter, the curve goes to higher TPR for low FPR as compared to the other parameter.

\begin{tabular}{cccc}
\hline \hline Parameter & AUC & ACC $(\%)$ & MCC \\
\hline$H^{2}$ & 0.651 & 73.91 & 0.512 \\
$H_{c 90}^{2}$ & 0.827 & 80.30 & 0.607 \\
$\int H^{2}$ & 0.863 & 79.71 & 0.596 \\
\hline
\end{tabular}

Table 4. Comparison of ROC curves for labor prediction.

\subsection{Discussion}

By using a basic classifier, we have shown that the two new measures, $H_{c 90}^{2}$ and $\int H^{2}$, give better results than the original measure $H^{2}$ for the detection of labor on monkey. The performances of the two parameters are very close to each other. The use of $\int H^{2}$ seems appealing since the computation time required for this parameter is very low. The complementarity of these two approaches on EHG has to be evaluated in order to see if we can increase the prediction ratio. We used as a first step a linear classifier based on only one parameter. If we choose to combine several parameters, classifier like neural network or support vector machine classifiers might give better performances than classical linear ones using only one parameter at a time. To demonstrate this, two examples of support vector machine classifiers, using both $H_{c 90}^{2}$ and $j H^{2}$, are illustrated Figure 14. They give better results than the previously obtained ones whatever the kernel used. The results obtained with a first order polynomial kernel are $\{\mathrm{ACC}=81.82$; $\mathrm{MCC}=0.639\}$ and with a second order polynomial kernel $\{\mathrm{ACC}=87.88$; $\mathrm{MCC}=0.769\}$. This first attempt of combining both synchronization parameters, described here, shows that each parameter seems to carry, at least in part, different information about the relationship between both channels. This fact is mainly noticeable during pregnancy. 

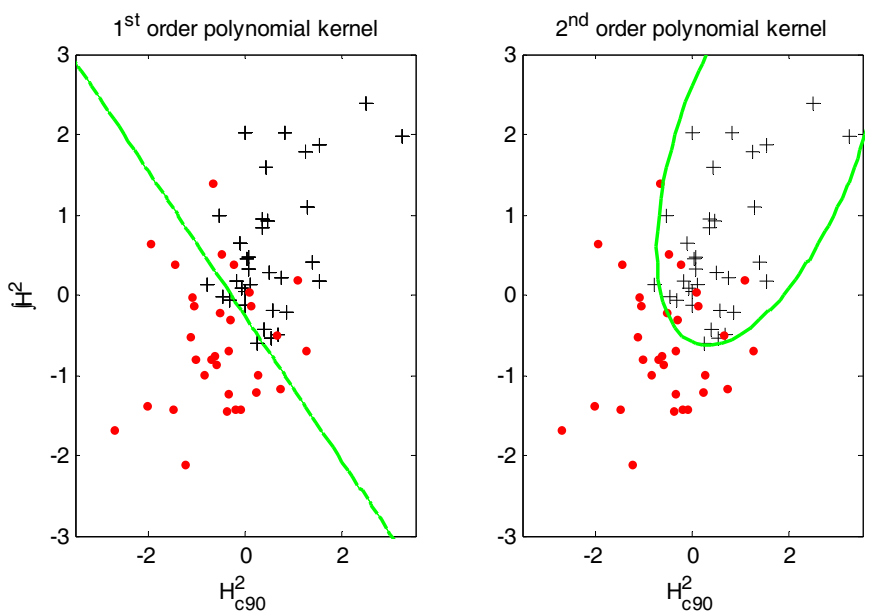

Fig. 14. Decision function (continuous line) obtained with a support vector machine parameterized by a $1^{\text {st }}$ order polynomial (left) or a $2^{\text {nd }}$ order polynomial kernel (right) trained on pregnancy (dot) and labor (cross) contractions dataset (normalized to have zero mean and unit variance on both components).

We have seen that taking into account nonlinearity and non stationarity in the EHG allows the prediction of labor with good accuracy. In the context of this work, we can only speculate as to what are the physiological origins of the observed changes, which occurred between pregnancy and labor. The increase in non-linearity, already observed (Radhakrishnan \& al., 2000), as well as in non stationarity might be explained by different phenomena like:

- $\quad$ The changes in the shape of the uterine cells action potential associated with the increase in the number of cell active at the same time. The action potential during pregnancy presents a lower bursting frequency thus less complexity than during labor. The increase in the number of active cells may result in an increase in the interferences pattern between the action potential of cells that are not synchronized, increasing thus non stationarity.

- The evolution of the propagation. It is well admitted that the propagation of the electrical activity of the uterus increases as labor approaches. This might results in more synchronized active cells. It is probably the case at a local scale mainly due to the increase in the number of GAP junction close to delivery (Garfield \& Hayashi, 1981; Blennerhassett \& Garfield, 1991). The propagation is however also supported at a bigger scale by other structures like the bundles or the different orientation of the uterine muscle fibers. These complex structures that also evolve during gestation, might also modify the characteristics of the recorded signals.

We do believe that only a physiological mathematical model of the electrical activity of the uterus taking into account the specificity and complexity of the uterine contractility may answer clearly these open questions on the physiology of the uterine muscle.

The promising results obtained here are on signals from a single monkey and have to be tested on human EHG. This may have relevance in helping to solve a real public health problem, namely preterm labor. 


\section{Conclusion}

Synchronization analysis methods are very powerful tools used to detect any coupling between two or more systems. All these methods suffer however from being very sensitive to the properties of the analyzed signals that may induce strong bias in the measure. The sources of bias might be simply experimental, like noise, or intrinsic to the signals of interest like non stationarity. In this chapter, we described the effects of these sources of bias on the synchronization analysis of synthetic as well as real EHG signals. We then proposed some general solutions rid the synchronization measures of bias. We finally defined two "new" synchronization measures that we used for analyzing the synchronization of the uterus during contractions.

The first method proposed consists in isolating the synchronization carried only by nonlinear components of a signal by using surrogate data analysis. This powerful method allowed us to differentiate to types of contractions: pregnancy and labor contractions. It permitted us then to propose a synchronization measure for labor prediction. The origins of this difference in terms of signal, as well as of physiological phenomenon, are still unknown but might be due partly to the increase in non stationarity of the EHG as labor approaches. The second method uses a segmentation algorithm to segment stationary parts of the signals and thus to avoid a compromise between the analysis window length and stationarity assumption. In addition to the absence of this need of a trade-off between these two important factors, the proposed method, which is based on the non stationarity properties of both signals, thus improves a previously proposed method that uses only one of the signals for the segmentation. This general method can be used with any synchronization measures. It allowed us to highlight the synchrony noticeable within EHG bursts. We were able to define another synchronization measure, defined as the integral of the obtained synchronization profile.

We finally showed that the two synchronization measures proposed by correcting the original nonlinear correlation coefficient, $\mathrm{H}^{2}$, are able to show an increase of the synchronization of the uterus during labor, when compared to pregnancy. Both parameters have nearly the same prediction performance with an accuracy of nearly $80 \%$. The original measure, $\mathrm{H}^{2}$, exhibited a decrease or at least no changes from pregnancy to labor, which would have lead to conclusion in contradiction with the physiology of the uterus. This is a typical example where a blind use of signal processing tools without taking into account possible sources of bias or limitation of the methods might lead to dramatic interpretation errors.

\section{Acknowledgments}

This work was supported by the Icelandic center of research, RANNIS, and the region Picardie-France in the project "Pôle périnatalité Enfance".

\section{References}

Ansari-Asl K., Senhadji L., Bellanger J.-J. \& Wendling F. (2006), "Time-frequency characterization of interdependencies in nonstationary signals: application to epileptic EEG," IEEE Trans Biomed Eng, vol. 52, pp. 1218-1226. 
Baccala L.A. \& Sameshima K. (2001a), "Partial directed coherence: a new concept in neural structure determination," Biol Cybern, vol. 84, pp. 463-74.

Baccala L.A. \& Sameshima K. (2001b), "Overcoming the limitations of correlation analysis for many simultaneously processed neural structures," Prog Brain Res, vol. 130, pp. 33-47.

Blanco S., Garcia H., Quiroga R.Q., L. Romanelli, and O. A. Rosso (1995), "Stationarity of the EEG Series," IEEE Engineering in Medicine and Biology Magazine, vol. 14, pp. 395-399.

Blennerhassett M.G. \& Garfield R.E. (1991), "Effect of gap junction number and permeability on intercellular coupling in rat myometrium," Am J Physiol, vol. 261, pp. C1001-9.

Borgnat P. \& Flandrin P. (2009), "Stationarization via surrogates," Journal of Statistical Mechanics: Theory and Experiment, vol. 2009, pp. P01001.

Buhimschi C., Boyle M.B. \& R. E. Garfield (1997), "Electrical activity of the human uterus during pregnancy as recorded from the abdominal surface," Obstet Gynecol, vol. 90, pp. 102-11.

Carré P. \& Fernandez C. (1998), "Research of stationary partitions in nonstationary processes by measurement of spectral distance with the help of nondyadic Malvar's decomposition," presented at IEEE-SP International Symposium on TimeFrequency and Time-Scale Analysis, Pittsburgh, PA, USA.

Devedeux D., Marque C., Mansour S., Germain G. \& Duchene J. (1993), "Uterine electromyography: a critical review," Am J Obstet Gynecol, vol. 169, pp. 1636-53.

Duchêne J., Marque C. \& S. Planque (1990), "Uterine EMG signal: Propagation analysis," presented at the Annual International Conference of the IEEE EMBS.

Euliano T.Y., Marossero D., Nguyen M.T., Euliano N.R., Principe J. \& R. K. Edwards (2009), "Spatiotemporal electrohysterography patterns in normal and arrested labor," Am J Obstet Gynecol, vol. 200, pp. 54 e1-7.

Garfield R.E. \& Hayashi R. H. (1981), "Appearance of gap junctions in the myometrium of women during labor," Am J Obstet Gynecol, vol. 140, pp. 254-60.

Garfield R.E., Maul H., Shi L., Maner W., Fittkow C., Olsen G. \& Saade G.R. (2001), "Methods and devices for the management of term and preterm labor," Ann $N$ Y Acad Sci, vol. 943, pp. 203-24.

Garfield R.E. \& Maner W.L. (2007), "Physiology and electrical activity of uterine contractions," Semin Cell Dev Biol, vol. 18, pp. 289-95.

Karlsson B., Terrien J., Guðmundsson V., Steingrímsdóttir T. \& Marque C. (2007), "Abdominal EHG on a 4 by 4 grid: mapping and presenting the propagation of uterine contractions," presented at 11th Mediterranean Conference on Medical and Biological Engineering and Computing, Ljubljana, Slovenia.

Kus R., Kaminski M. \& Blinowska K.J. (2004), "Determination of EEG activity propagation: pair-wise versus multichannel estimate," IEEE Trans Biomed Eng, vol. 51, pp. 150110.

Leman H. \& Marque C. (2000), "Rejection of the maternal electrocardiogram in the electrohysterogram signal," IEEE Trans Biomed Eng, vol. 47, pp. 1010-7.

Leman H., Marque C. \& Gondry J. (1999), "Use of the electrohysterogram signal for characterization of contractions during pregnancy," IEEE Trans Biomed Eng, vol. 46, pp. 1222-9. 
Maner W.L. \& Garfield R.E. (2007), "Identification of human term and preterm labor using artificial neural networks on uterine electromyography data," Ann Biomed Eng, vol. 35, pp. 465-73.

Pereda E., Quiroga R. Q. \& Bhattacharya J. (2005), "Nonlinear multivariate analysis of neurophysiological signals," Progress in Neurobiology, vol. 77, pp. 1-37.

Pijn J.P., Vijn P.C., Lopes da Silva F.H., Van Ende Boas W. \& Blanes W. (1990), "Localization of epileptogenic foci using a new signal analytical approach," Neurophysiol Clin, vol. 20, pp. 1-11.

Prichard D. \& Theiler J. (1994), "Generating surrogate data for time series with several simultaneously measured variables," Physical Review Letters, vol. 73, pp. 951-954.

Quian Quiroga R., Kreuz T. \& Grassberger P. (2002), "Event synchronization: a simple and fast method to measure synchronicity and time delay patterns," Phys Rev E Stat Nonlin Soft Matter Phys, vol. 66, pp. 041904.

Radhakrishnan N., Wilson J. D., Lowery C., Murphy P. \& Eswaran H. (2000), "Testing for nonlinearity of the contraction segments in uterine electromyography," International Journal of Bifurcation and Chaos, vol. 10, pp. 2785-2790.

Ramon C., Preissl H., Murphy P., Wilson J.D., Lowery C. \& Eswaran H. (2005), "Synchronization analysis of the uterine magnetic activity during contractions," Biomed Eng Online, vol. 4, pp. 55.

Schreiber T. \& Schmitz A. (2000), "Surrogate time series," Physica D, vol. 142, pp. 346-382.

Terrien J., Germain G. \& Marque C. (2008a), "Ridge extraction from the time-frequency representation (TFR) of signals based on an image processing approach: Application to the analysis of uterine electromyogram AR TFR," IEEE Trans Biomed Eng, vol. 55, pp. 1496-1503.

Terrien J., Hassan M., Marque C. \& Karlsson B. (2008b), "Use of piecewise stationary segmentation as a pre-treatment for synchronization measures," presented at 30th Annual International Conference of the IEEE EMBS, Vancouver, Canada.

Young R.C. \& Hession R.O. (1999), "Three-dimensional structure of smooth muscle in termpregnant human uterus," Obstet Gynecol, vol. 93, pp. 94-99.

Young R.C. (1997), "A computer model of uterine contractions based on action potential propagation and intercellular calcium waves," Obstet Gynecol, vol. 89, pp. 604-8.

Wendling F., Bartolomei F., Bellanger J.J. \& Chauvel P. (2001), "Interpretation of interdependencies in epileptic signals using a macroscopic physiological model of the EEG," Clin Neurophysiol, vol. 112, pp. 1201-18. 


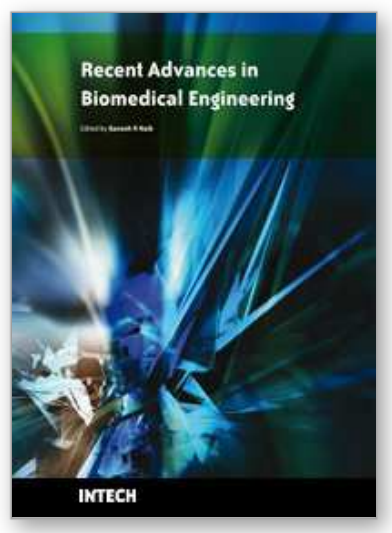

\author{
Recent Advances in Biomedical Engineering \\ Edited by Ganesh R Naik
}

ISBN 978-953-307-004-9

Hard cover, 660 pages

Publisher InTech

Published online 01, October, 2009

Published in print edition October, 2009

The field of biomedical engineering has expanded markedly in the past ten years. This growth is supported by advances in biological science, which have created new opportunities for development of tools for diagnosis and therapy for human disease. The discipline focuses both on development of new biomaterials, analytical methodologies and on the application of concepts drawn from engineering, computing, mathematics, chemical and physical sciences to advance biomedical knowledge while improving the effectiveness and delivery of clinical medicine. Biomedical engineering now encompasses a range of fields of specialization including bioinstrumentation, bioimaging, biomechanics, biomaterials, and biomolecular engineering. Biomedical engineering covers recent advances in the growing field of biomedical technology, instrumentation, and administration. Contributions focus on theoretical and practical problems associated with the development of medical technology; the introduction of new engineering methods into public health; hospitals and patient care; the improvement of diagnosis and therapy; and biomedical information storage and retrieval. The book is directed at engineering students in their final year of undergraduate studies or in their graduate studies. Most undergraduate students majoring in biomedical engineering are faced with a decision, early in their program of study, regarding the field in which they would like to specialize. Each chosen specialty has a specific set of course requirements and is supplemented by wise selection of elective and supporting coursework. Also, many young students of biomedical engineering use independent research projects as a source of inspiration and preparation but have difficulty identifying research areas that are right for them. Therefore, a second goal of this book is to link knowledge of basic science and engineering to fields of specialization and current research. The editor would like to thank the authors, who have committed so much effort to the publication of this work.

\title{
How to reference
}

In order to correctly reference this scholarly work, feel free to copy and paste the following:

Terrien Jeremy, Marque Catherine, Germain Guy and Karlsson Brynjar (2009). Sources of Bias in Synchronization Measures and how to Minimize Their Effects on the Estimation of Synchronicity: Application to the Uterine Electromyogram, Recent Advances in Biomedical Engineering, Ganesh R Naik (Ed.), ISBN: 978953-307-004-9, InTech, Available from: http://www.intechopen.com/books/recent-advances-in-biomedicalengineering/sources-of-bias-in-synchronization-measures-and-how-to-minimize-their-effects-on-theestimation-of-s

\section{INTECH}

open science | open minds

InTech Europe

InTech China

www.intechopen.com 
University Campus STeP Ri Slavka Krautzeka 83/A

51000 Rijeka, Croatia

Phone: +385 (51) 770447

Fax: +385 (51) 686166

www.intechopen.com
Unit 405, Office Block, Hotel Equatorial Shanghai No.65, Yan An Road (West), Shanghai, 200040, China 中国上海市延安西路65号上海国际贵都大饭店办公楼 405 单元

Phone: +86-21-62489820

Fax: +86-21-62489821 
(C) 2009 The Author(s). Licensee IntechOpen. This chapter is distributed under the terms of the Creative Commons Attribution-NonCommercial-ShareAlike-3.0 License, which permits use, distribution and reproduction for non-commercial purposes, provided the original is properly cited and derivative works building on this content are distributed under the same license. 